\title{
Technical Terms of the Category Locus and Their Semantic Relations within a Terminology
}

\author{
Maxim Latu ${ }^{1} \&$ Alina Levit ${ }^{2}$ \\ ${ }^{1}$ Ph.D. in Philology, Associate Professor of the Western European Languages and Cultures \\ Department, Head of the Research and Educational Center "Applied Linguistics, \\ Terminology Studies and Linguocognitive Technologies”, Pyatigorsk State University, \\ Pyatigorsk, Russia. E-mail: laatuu@yandex.ru \\ ${ }^{2}$ Researcher at the Research and Educational Center "Applied Linguistics, Terminology \\ Studies and Linguocognitive Technologies”, Pyatigorsk State University, Pyatigorsk, Russia. \\ E-mail: indfund@mail.ru.
}

Received November 10, 2016; Revised February 15, 2017; Accepted March 17, 2017; Published May 7, 2017.

\begin{abstract}
The article focuses on the space research technical terms of the category Locus and sets of adjacent technical terms in fragments of the terminological network. We argue that the technical terms of the category Locus have the potential to establish semantic relations of certain types with the technical terms of certain categories only, thus forming prototypic semantic schemes. We consider the categories of the defined first and second order technical terms and the types of semantic relations between them. The productive and non-productive semantic relations as well as those that are not characteristic of the category Locus are analyzed.
\end{abstract}

Keywords: technical term, concept, referent, category, semantic network, semantic relation, knowledge representation, definition

\section{Introduction}

A semantic network is considered to be an important tool of knowledge representation and visualization. As R. Hartley and J. Barnden (1997) say, it has led dual existence "as a motivational diagrammatic form of knowledge representation, on the one hand", and "as an internal, computerized form, suitable for a variety of computational methods", on the other. Consisting of vertices and arcs, a semantic network "can keep all the information about an entity at a single node and show related information by arcs connected directly to that node" (Sowa, 1987). In other words "while concepts are the building blocks of knowledge, relations act as the cement that links up concepts into knowledge structures" (Khoo \& Jin-Cheon Na, 2006). Two vertices appear to be adjacent if there is a relation that connects them (Borge-Holthoefer \& Arenas, 2010), a vertex with its adjacent vertices form a neighborhood structure (Steyvers \& Tenenbaum, 2004). In this respect semantic networks have a lot in common with ontologies though A-B. M. Salem and M. Alfonse (2008) focus on the differences that exist between them. B. Harrington (2010) points to the fact that semantic networks are very useful for determining and studying semantic relatedness of terms. The types of semantic relations within a semantic network may differ depending on the

(c) AesthetixMS 2016. This Open Access article is published under a Creative Commons Attribution Non-Commercial 4.0 International License (http://creativecommons.org/licenses/by-nc/4.o/), which permits non-commercial re-use, distribution, and reproduction in any medium, provided the original work is properly cited. For citation use the DOI. For commercial re-use, please contact editor@rupkatha.com. 
objectives (Cerban, 2010), the concepts that constitute the vertices and the sphere that is modelled. Semantic networks may be based on different principles, for example, synsets and hyponymy relations as in the case of WordNet (Fellbaum, 1998). We propose that semantic networks can also be used to study systemic relations within terminologies that are considered to be complex semiotic systems of interlinked signs (Kukhno, 2014; Staicu \& Wessels, 2010). Thus, a terminological network is a kind of semantic network the vertices of which are represented by technical terms while arcs represent semantic relations between them.

This study is devoted to the peculiarities of organization of knowledge expressed by the technical terms of different categories within the terminology of space research. Based on the results of the previous studies, we hypothesized that the types of semantic relations that a technical term establishes with the adjacent technical terms as well as the categories they represent largely depend upon the category this technical term refers to. Thus, each category of technical terms is characterized by prototypic semantic schemes (a certain category linked by a definite type of semantic relation to a certain category). These may diverge for different categories of technical terms and be of various productivity. In the paper we focus on the specificity of the technical terms of the category Locus and the semantic relations with the technical terms of some other categories that they have the potential to establish.

\section{Methodology and research algorithm}

\subsection{Previous research and approaches}

Despite the relevance and topicality, different issues concerning the nature, the specificity and characteristics of structure of terminological networks still remain unexplored. The works of M.G. Malkovskiy and S.Y. Solovyev (2012) significantly contributed to the studies as the researchers tried to solve applied problems of using terminological networks for web glossaries development. They tried to integrate the terminological networks of different fields of knowledge into a single network that they call a universal terminology space (UTS) (Malkovskiy \& Solovyev, 2012). Their approach dictates that terminological networks organized in a UTS represent a complex structure where the vertices are technical terms linked up by two types of binary relations: "it is" (which corresponds to ISA and AKO semantic relations) and "refers to" that incorporates all the other types of semantic relations (Malkovskiy \& Solovyev, 2003). It is noted that, as in any ordinary semantic network, a node can have an arbitrary number of semantic relations (Malkovskiy \& Solovyev, 2004a). According to the point of view of the researchers, polysemantic technical terms represent a special case of structural elements, because they refer to several vertices (Malkovskiy \& Solovyev, 2004b). Thus, a terminological network is a "natural superstructure based on a multitude of definitions of technical terms" (Malkovskiy \& Solovyev, 2014).

I. Atanasova and S. Nakov (2003) developed ArtsSemNet that is an electronic glossary of technical terms of fine art based on the network principles of their organization. The vertices a mainly linked by hyponymy (AKO - "a kind of") relations, however relations based on synonymy, homonymy, polysemy are also represented. The researchers remark that ArtsSemNet does not detect and display hyponyms that refer to one and the same generic term and treats meronyms as hyponyms. D.V. Lande (2014) suggests an alternative method of automatic construction of a network of natural hierarchies of technical terms (NNHT) based on the identification of technical terms in texts, where "all links are determined by the natural use of the words and phrases and correspond to the occurrence of terms into the other ones". In other words the relations between the vertices merely reflect the combinatory power and the derivative processes when one 
technical term is used in the structure of a larger multicomponent technical term. I.O. Serdceva (2006) constructs a terminological network of computer science technical terms that are also linked only by hyponymy semantic relation. The considered approaches to the construction of the terminological networks are mostly based on hyponymy relations without specifying other possible types and paying attention to differentiation of vertices that we see as a disadvantage.

\subsection{Procedure and data analysis}

This research is generally based on the principles of semantic network analysis applied to the study of organization of technical terms within a terminology, as well as the approaches developed by M.G. Malkovskiy, S.Y. Solovyev (2003), I. Atanasova, S. Nakov (2003) and others discussed earlier as the technical terms of a definite branch represent the vertices interrelated to a certain number of adjacent technical terms in each case. However our approach to terminological network construction is based on the differentiation between the types of vertices as well as a more detailed division of the systemic semantic relations. Classification of vertices into types reflects the natural stratification of technical terms within a terminology according to the categories of concepts that they express (Grinev-Grinevich, 2008). Their referents may be material and non-material. The material referents may be of natural origin that constitute such categories as Natural object/phenomenon (objects and phenomena of the physical world, e.g. variable star), Natural substance (substances of natural origin, e.g. plasma), Locus (regions and spatial extents, e.g. interplanetary space) and man-made among which are Mechanism (units of equipment, e.g. solar radio telescope), Instrument (tools and products, e.g. sextant), Material (man-made materials, e.g. glass-fibre reinforced plastic), Construction/man-made locus (e.g. orbital platform). The non-material constitute such categories as Process, Characteristic, Actor, Situation and Ideal phenomenon (that is an image or an abstraction necessary for further academic knowledge development in the field, e.g. Big Bang model, light year, etc.) (Latu, 2015). The semantic relations between them are subdivided into the following types: $A K O$ - "a kind of" (the relation that links the generic term and its direct hyponyms), ISA - "is a" (the relation that expresses inclusion of the referent into some aggregate that is not the direct generic term or concurrence), At - "attribute" (the relation with characteristics and properties), $P O$ - "part of" (the relation that links meronyms and holonyms), $A g$ - "agent" (the relation that links the initiator or doer with the process), Obj "object" (the relation between the process and a referent under its influence), $S r$ - "source" (a relation between the initial position and a referent), Rec - "recipient" (a relation between a referent and its final position), Inst - "instrument" (a relation with the referent that is considered to be a means or an implement in pursuing some aim), $R$ - "result" (cause and effect relation), Loc - "locus" (a relation that shows the exact location), Eop - "explicit opposition" (a relation between hyponyms of one generic concept the antonymous nature of which is reflected in the inner form of their technical terms), Iop - "implicit opposition" (a relation between hyponyms of one generic concept the antonymous nature of which is not reflected in the inner form of their technical terms), Sub - "subordinate" (a relation that links the referents belonging to the category Actor: superiors and subordinates), a number of relations based on prepositions such as Inter, etc. (Latu, 2016).

As the first step, the technical terms that express the category Locus were extracted from the body of the specialized vocabulary of astrophysics and space research (totally 3144 technical terms) that we have previously assembled from specialized literature and Internet sources, systemized according to the nature and peculiarities of the referents they express and classified

into the categories that were mentioned above. The number of the extracted vocabulary units that 
express the concepts of the analyzed category constituted 227 in the given sample. Then several definitions for each technical term of the considered category were collected from different specialized sources (usually no less than 3) as well as fragments of texts where it appears and interacts with other technical terms of this field of knowledge. The whole corpus constituted 685 analyzed definitions and more than 900 studied text extracts. At the next stage these definitions were carefully analyzed and the adjacent technical terms that are used to define each of the technical terms of the category Locus were detected, grouped and qualified as the first order adjacent technical terms. All the adjacent technical terms that do not appear in the definitions but are still related to the analyzed technical term of the category Locus are qualified as the second order. Similarly, these were detected in the specialized texts and grouped for each of the technical terms that express the category Locus.

As the third step of the study, the types of semantic relations between each of the analyzed technical terms of the considered category and the adjacent technical terms that belong to different categories were defined and fragments of the semantic network were constructed. As we have already mentioned, in the terminological network the vertices are represented by the technical terms and their concepts, the types of vertices correspond to the categories and the arcs represent types of the semantic relations between them. Then the comparative analysis and the statistical study were applied. The types of arcs and the adjacent vertices within the fragments were compared and counted to define what types of semantic relations are productive and nonproductive for the category Locus, what categories the adjacent technical terms of both the first and second order refer to, what prototypic semantic schemes based on their relations are characteristic of the considered category.

\section{Results}

The results of the analysis prove the hypothesis that certain categories of concepts expressed by technical terms establish prototypic semantic schemes of interaction with other adjacent concepts of the same field of knowledge. The category Locus encompasses all technical terms that express the academic concepts representing spatial extents, regions, dimensional areas defined within a certain field of knowledge. As the study of the semantic network of the terminology of space research have demonstrated, the technical terms that belong to the category Locus are linked up to adjacent technical terms of certain categories only by means of certain semantic relations. These appear to be of different productivity and frequency of manifestation for the considered category (some semantic relations turn to be very productive while a definite number of others are not, some semantic relations are not present at all). The analysis of definitions helped to define the adjacent technical terms of the first order and the categories they belong to as well as the types of semantic relations between them.

\subsection{PO and AKO semantic relations}

Within the terminology of space research there is a number of concepts that come within the category, for example, solar corona, interstellar space, Cassini division, etc. that is clearly seen from their definitions. Thus, the technical term solar corona is defined as "the outermost region of the sun's atmosphere; visible as a white halo during a solar eclipse" (Lieberman, 2014,), "outermost region of the Sun's atmosphere, consisting of plasma (hot ionized gas)" (Anderson, 2011), "Corona is the outer part of the Sun's atmosphere and is the region where prominences appear. Prominences are immense clouds of glowing gas that erupt from the upper Chromosphere. The outer region of the 
corona stretches far into space (millions of $\mathrm{kms}$ ) and consist of particles traveling slowly away from the Sun. Along with the Chromosphere, Corona is only visible during total solar eclipse, when the Sun's surface is completely hidden behind the Moon" (Sreepat, 2013). These definitions suggest the solar corona is the outer part of the sun's atmosphere that also refers to the category Locus, because like the atmosphere of any star it represents the region in space: "a stellar atmosphere is a transition region from the stellar interior to the interstellar medium" (Gray, 2005). "Rather, our picture seems to be evolving toward an atmosphere that is a transition region between the stellar interior and the interstellar medium" (Jager, 2012). As shown in figure 1 , these two technical terms belong to the same category linked by PO semantic relation because both concepts are places in space of specific extent and boundaries that the following passages also make clear: "the solar atmosphere, the exterior of the Sun, is composed of the photosphere, chromosphere, and the corona" (Alma; Ghassemi \& Foster, 2009). "The Sun's outer visible layer is called the Photosphere which has a spotted appearance due to the continuous and turbulent eruptions of energy from its surface. The Photosphere is the visible energy surface of the Sun, where temperatures reach $5,500{ }^{\circ} \mathrm{C}$ " (Sreepat, 2013). The defined concept is also linked by PO semantic relation with the technical term solar eclipse which represents the category Situation because it is one of the integral notions that constitute this astronomical event. Likewise, the technical terms interstellar space is related to outer space by means of PO semantic relation being a part of it. There are also other technical terms that are related to the solar corona by the same relation but not mentioned in the definitions. Thus, the technical term coronal hole that also represents the category Locus is considered to be a large dark region that is a part of solar corona. Similarly, as the technical terms Huygens gap and Laplace gap represent dark areas within a larger region between A ring and B ring of Saturn expressed by the technical term Cassini division, they are linked to it by PO semantic relation. "The famous Cassini Division (the largest dark gap) in the rings is clearly visible, as is a thin division near the edge of the ring system. This is the Encke Division, which has never been photographed from the Earth" (Brandt \& Petersen, 1998). With regard to the analyzed category PO semantic relation proves to be one of the most productive and amounts to about $22 \%$ among the others.

It is worth noting that AKO semantic relation is not typical of the category Locus and does not appear in the definitions unlike for the category Natural object the technical terms of which establish complex hierarchical classifications (i.e. radio pulsar is a kind of pulsar that is a kind of neutron star which in turn is a kind of star, etc.). This happens because there may not be a direct generic concept that is expressed by a technical term which belongs to the same field of knowledge. Instead words of general use may appear in definitions such as area, region, layer, etc. that are linked to the defined term by ISA ("is a") semantic relation. But they are not reflected in terminological network since they are not technical terms. This situation makes the considered category comparable to the categories Process and Characteristic for which the AKO semantic relation is also unproductive (however unlike the category Locus it is more common for them to establish small classifications). Similarly, a concept that expresses the category Locus may not have hyponyms, for example, interplanetary space, habitable zone, etc. have no subcategories. The technical term convective zone comes as an exception as there are hyponyms core convective zone and outer convective zone. The technical term orbit is a representative of the category Ideal phenomenon (usually defined by the word path or trajectory) however its referent sometimes may be considered as a quasi-area or place. It does not have a generic technical term, but is subdivided into numerous types that form different classifications. Among these are Low Earth orbit which is a kind of geocentric orbit with altitudes of 100 to 1240 miles, lunar orbit, disposal orbit, where 
spacecraft are placed at the end of their operational life, etc. as opposed to non-inclined orbit, synchronous orbit, parabolic orbit that are not seen as places at all.

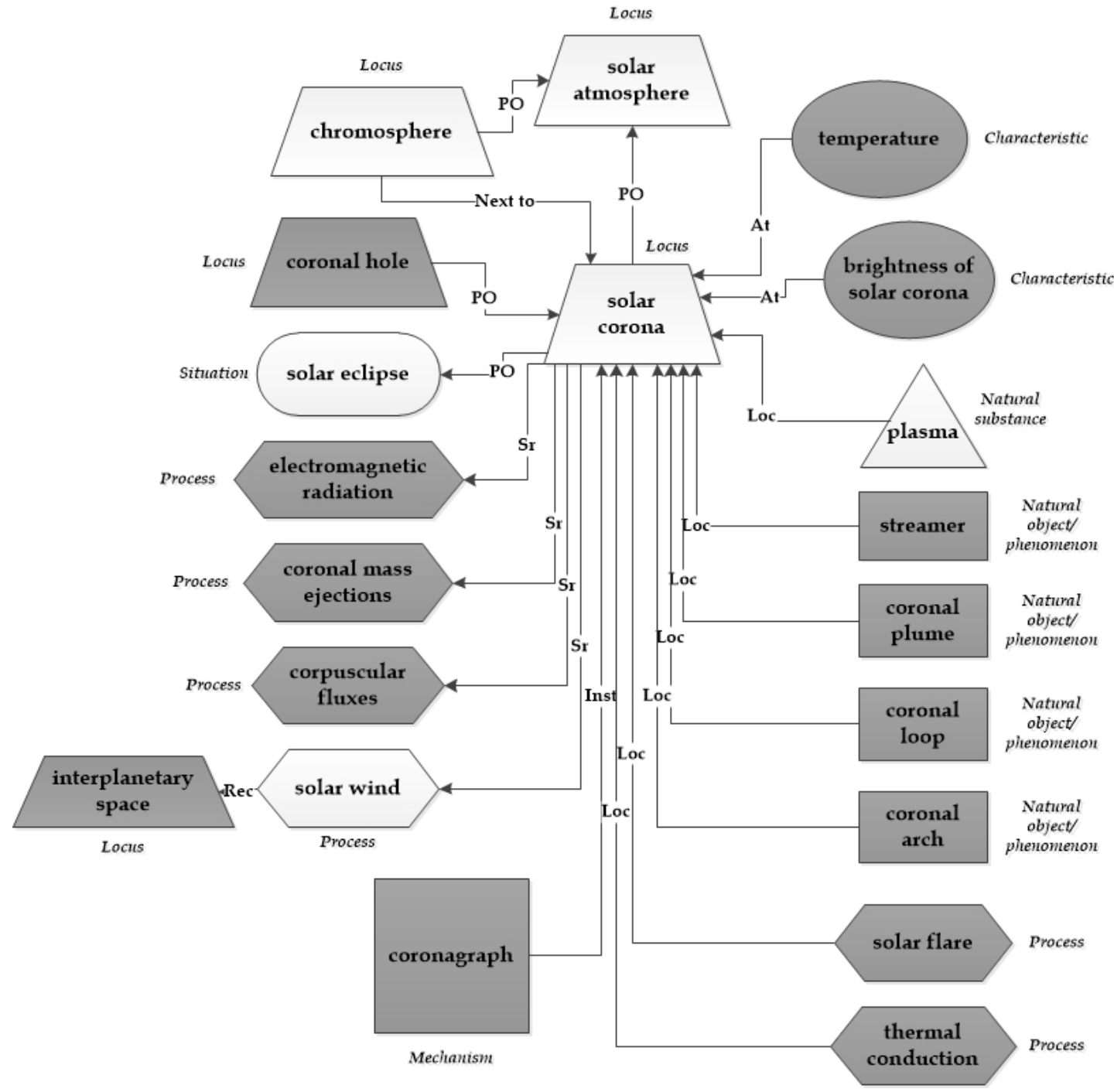

Figure 1. A fragment of the terminological network. Semantic relations that link the technical term solar corona with some adjacent technical terms

\subsection{At semantic relation}

Generally speaking, the number of adjacent technical terms of the second order (extracted from texts) is no less than thrice as much as the quantity of technical terms of the first order (extracted from definitions) for each considered technical term. The technical term solar corona is not an exception. It is also related to a number of technical terms that express the category Characteristic. Thus, the technical term temperature is related to the solar corona by means of At semantic relation. "Parker's key insight was that high temperatures in the corona can provide enough energy per particle to produce a natural transition from a subsonic (bound, negative total energy) state near the Sun to a supersonic (outflowing, positive total energy) state in interplanetary space." (Cranmer, 2002); "The key to understanding the heating and acceleration of the solar wind is to determine what mechanisms create the decidedly non-Maxwellian distributions of the electrons 
and ions. If the high temperatures of the corona can be explained with a theory that uses nonMaxwellian distributions, explicit coronal heating mechanisms such as via waves may be unnecessary, but such a theory relegates the solar wind acceleration problem to what causes these non-Maxwellian distribution" (Russell, 200o). Likewise, it is linked to the technical term brightness of the solar corona. "The brightness of the solar corona has long been known to consist of mainly two components, i.e., $K$ - and F-corona, although, as a further component, emission lines of highly charged coronal ions also influence the coronal brightness" (Kimura \& Mann, 1998), "The appearance of the solar corona during a total solar eclipse. The corona is extremely faint relative to the visible disk of the Sun, having a maximum brightness ratio of $\sim 10^{-9}$ within a single solar diameter away from the visible limb. On a good day at ground level the sky brightness exceeds that of the corona by 3 to 5 orders of magnitude, so that the corona is completely invisible to the naked eye" (Golub \& Pasachoff, 2010). "The total brightness of the corona as measured between $r=1.03$ and 6.0 varies from sunspot minimum to maximum by about a factor 2 (2,3)" (Evans, 2013). These concepts characterize the solar corona and are mentioned in specialized texts, but do not appear in definitions. In this respect At semantic relation is far not so productive and constitutes less than $7 \%$.

\subsection{Loc semantic relation and the categories of the adjacent technical terms}

It is worth noting in this respect that very often a certain region is closely related and associated with its constituents that fulfill it. The technical terms interstellar space and interstellar medium as well as interplanetary space and interplanetary medium are sometimes considered as synonyms: "A spacecraft that passes through the boundary between interplanetary space and true interstellar space - the region between stars that is occupied by the interstellar medium - and that possibly travel to the vicinity of other stars" (Darling \& Schulze-Makuch, 2016). However the first ones represent the regions in space while the second point at all the matter that fills them: "the interstellar medium is the gas and dust that pervade interstellar space: the matter that exists between the star systems within a galaxy. It fills interstellar space and blends smoothly into the surrounding intergalactic space" (Esomba, 2012). This fact results into that some semantic relations that are established with the objects and phenomena within the space are extrapolated to the space itself. It is clear that the solar corona is hot because of the presence of plasma that is characterized by temperature. The constituent parts of interstellar medium among which are interstellar gas and interstellar dust are related to the term by PO semantic relation: "In astronomy, the interstellar medium (ISM) is the matter that exists in the space between the star systems in a galaxy. This matter includes gas in ionic, atomic, and molecular form, as well as dust and cosmic rays" (Anderson, 2015); "By mass, $99 \%$ of the ISM is gas in any form, and $1 \%$ is dust. Of the gas in the ISM, by number $91 \%$ of atoms are hydrogen and 9\% are helium, with $0.1 \%$ being atoms of elements heavier than hydrogen or helium" (Anderson, 2015), "The interstellar medium, on the other hand, is an extremely complex system in which it is not possible to describe the physical status of the matter and it is structural and dynamical configurations, as well as the radiation fields, with a restricted number of parameters; instead, a full and detailed account of the history of the system is required for such a description" (Habing \& Olofsson, 2013). Their adjacency to the technical term interstellar space is marked by Loc semantic relation.

It was discovered that Loc semantic relation is the most productive and amounts to $41 \%$. It links the category Locus with the largest number of other categories among which are Process, Natural Object, Substance, Ideal phenomenon, Construction, Mechanism. Thus, the solar corona is the location of plasma that is the technical term that refers to the category Substance: "in a hot 
tenuous plasma such as the corona the resistivity is extremely small, because collisions are rare, and the shear viscosity is small because the particles are tied to the magnetic field lines" (Golub \& Pasachoff, 2010). The relation with this technical term is reflected in one of the definitions which makes it a unit of the first order for the solar corona. However there are various natural objects and phenomena that are related to this and other regions in the Sun's atmosphere that are not mentioned in definitions but appear in scientific texts: "Active regions involve all the phenomena directly linked to the magnetic field, which occur at different heights on the Sun's surface: sunspots and faculae, happening in the photosphere, spicules, $\underline{H \alpha}$ filaments and plages in the chromosphere, prominences in the chromosphere and transition region, and flares and coronal mass ejections happening in the corona and chromosphere", "the corona displays a variety of features including streamers, plumes, and loops" (National Research Council, 2003). "The chromosphere can be very dynamic with hot jets of gas (spicules) extending high above the surface” (Moldwin, 2008).

\section{4. $\mathrm{Sr}$ and Rec semantic relations}

The category Locus can also be linked to the category Process by means of Loc, Sr and Rec semantic relations because a space extent may be the exact place where the action takes place, serve a source or the recipient of a process. It is worth noting that some processes may be general or specific to a particular place. Thus, electromagnetic radiation linked by $\mathrm{Sr}$ semantic relation to solar corona "The appearance of outer-space solar astronomy, along with advances in experimental technique and theoretical conceptions, led to the idea that the solar flare is a phenomenon peculiar to the solar atmosphere, which traverses all layers from the photosphere up to the corona and displays electromagnetic radiation in radiowaves ranging up to $y$-radiation and emits corpuscular fluxes and particles" (Sobelman, 1992), is also associated with other places or objects in space that are considered to be its source. "The corona emits energy of many different wavelengths that emerge from the interior of the Sun, from long wavelengths radio waves to short wavelengths $x$-rays" (Alma., Ghassemi., Foster, 2009, p. 29). The technical terms coronal mass ejection and solar wind linked to the technical term solar corona by means of the same semantic relation because the concepts they express are specific to the place or its constituents: "the interaction between the plasma and the magnetic field in the solar corona determines what kind of phenomena will occur in the corona. An unbalanced magnetic flux causes the magnetic field lines to open and a so called coronal hole is formed. From the coronal hole a fast stream of plasma (the solar wind) expands into the interplanetary space". "Coronal holes are the lowest density plasma components of the Sun's outer atmosphere, and are associated with rapidly expanding magnetic fields and the acceleration of the high-speed solar wind" (Cranmer, 2002). "Knowledge of the fast-slow solar wind boundaries in interplanetary space have, until the more recent polar passages of Ulysses, been derived from spacecraft observations carried out in or near the ecliptic plane, or from remote sensing measurements which can probe the solar wind at latitudes and distances not yet accessible by spacecraft" (Cranmer \& Kohl, 2013). Since the solar wind streams from the solar corona into interplanetary space, the technical terms interplanetary space and solar wind are linked together by Rec semantic relation. Sr semantic relation constitutes $7 \%$, while Rec semantic relation is about 6\%. The technical terms star formation (Process) and interstellar space (Locus) are linked by Loc semantic relation. The process gravitational attraction is linked to the concept Roche lobe by the same semantic relation since the latter is "a region of space surrounding two massive bodies revolving around each other under their mutual gravitational attraction, such that the gravitational attraction of each body dominates the lobe surrounding it". "Most gravitational systems are well approximated by an ensemble of point masses moving under their mutual gravitational attraction 
and range from planetary systems (such as our own) to star clusters, galaxies, galaxy clusters and the universe as a whole" (Dehnen \& Read, 2011).

\subsection{Inter, Inst and other semantic relations}

One of the features of the category Locus is the presence of a variety of relations based on prepositions, among which are inter, under, around, next to, etc. These are established between the technical terms of the category Locus or link the category Locus to the category Natural object, thus forming definite prototypic semantic schemes. The relations with particular technical terms of the categories may mark the borderlines of the considered region in space. The technical term Cassini division is related to the $A$ ring and $B$ ring of Saturn by Inter semantic relation as it lies in between them. In the studied termoinology these semantic relations, however, are still not so productive as Loc or PO semantic relations with Inter and Around being the most numerous. The Roche lobe is stated to be an area around a star within a binary system with the technical terms that express these concepts linked by Arnd and Loc semantic relations correspondently. Arnd semantic relation amounts to $6 \%$ and Inter semantic relation to $5 \%$ as shown in the table 1 below.

\begin{tabular}{|c|c|c|c|c|c|c|c|c|c|c|c|c|c|}
\hline Loc & $\mathrm{PO}$ & At & $\mathrm{Sr}$ & Rec & Arnd & Intr & $\begin{array}{l}\text { Abo } \\
\text { ve }\end{array}$ & $\begin{array}{l}\text { Und } \\
\text { er }\end{array}$ & $\begin{array}{l}\text { Ins } \\
\mathrm{t}\end{array}$ & $\begin{array}{l}\mathrm{Ne} \\
\mathrm{xt} \\
\text { to }\end{array}$ & $\begin{array}{l}\text { Be } \\
\text { hin } \\
\text { d }\end{array}$ & ISA & $\begin{array}{l}\text { AK } \\
\mathrm{O}\end{array}$ \\
\hline $\begin{array}{l}40.2 \\
\%\end{array}$ & $\begin{array}{l}22.3 \\
\%\end{array}$ & $7.2 \%$ & $\begin{array}{l}6.8 \\
\%\end{array}$ & $5 \cdot 7 \%$ & $5 \cdot 3 \%$ & $4.5 \%$ & $1.9 \%$ & $1.8 \%$ & $\begin{array}{l}1.1 \\
\%\end{array}$ & $\begin{array}{l}1.1 \\
\%\end{array}$ & $\begin{array}{l}0.8 \\
\%\end{array}$ & $\begin{array}{l}\text { o.7 } \\
\%\end{array}$ & $\begin{array}{l}\text { o.6 } \\
\%\end{array}$ \\
\hline
\end{tabular}

Table 1. The percentage ratio of the semantic relations with regard to the category Locus

The considered category can also be semantically related to the category Mechanism by means of Inst semantic relation. The semantic relation links the technical term solar corona with the technical terms that express different kinds of coronographs, Hi-C (high resolution coronal imager) that are used to study it: "the Sun's corona extends millions of kilometres into space and is most easily seen during a total solar eclipse, but it is also observable with a coronagraph" (Pasachoff, 2013). "The corona is briefly visible to the unaided eye during a total eclipse of the Sun, for at most 7.5 minutes; at other times it can be observed in visible white light by using a special instrument called a coronagraph. The visible light corona may be divided into the inner $K$ or electron corona, with a continuous spectrum, and the outer $F$ or dust corona that displays Fraunhofer absorption lines" (Lang, 2007). "The Ultraviolet Coronagraph Spectrometer (UVCS) aboard the Solar and Heliospheric Observatory (SOHO) spacecraft has revealed surprisingly large temperatures, outflow speeds, and velocity distribution anisotropies for positive ions in coronal holes. We review recent observations, modeling techniques, and proposed heating and acceleration processes for protons, electrons, and heavy ions" (Cranmer, 2002). Inst relation may also link technical terms of the category Mechanism with various phenomena associated with the concept of the category Locus as we pointed at earlier. At the same time there may not be a specific device and a corresponding technical term to study only a particular area in space, for example, there is no specific equipment to observe and explore the Huygens gap solely. "The Huygens gap separates the B ring, whose outer edge is defined by the strong Mimas 2:1 inner Lindblad resonance, from the Cassini Division proper. It is the broadest gap in Saturns rings, being $25 \%$ wider than the Encke Gap in the outer A ring" (Dougherty., Esposito., Krimigis, 2009). The figure 2 below illustrates the 
results of the comparative and statistical analyses of the patterns of the technical terms interaction.
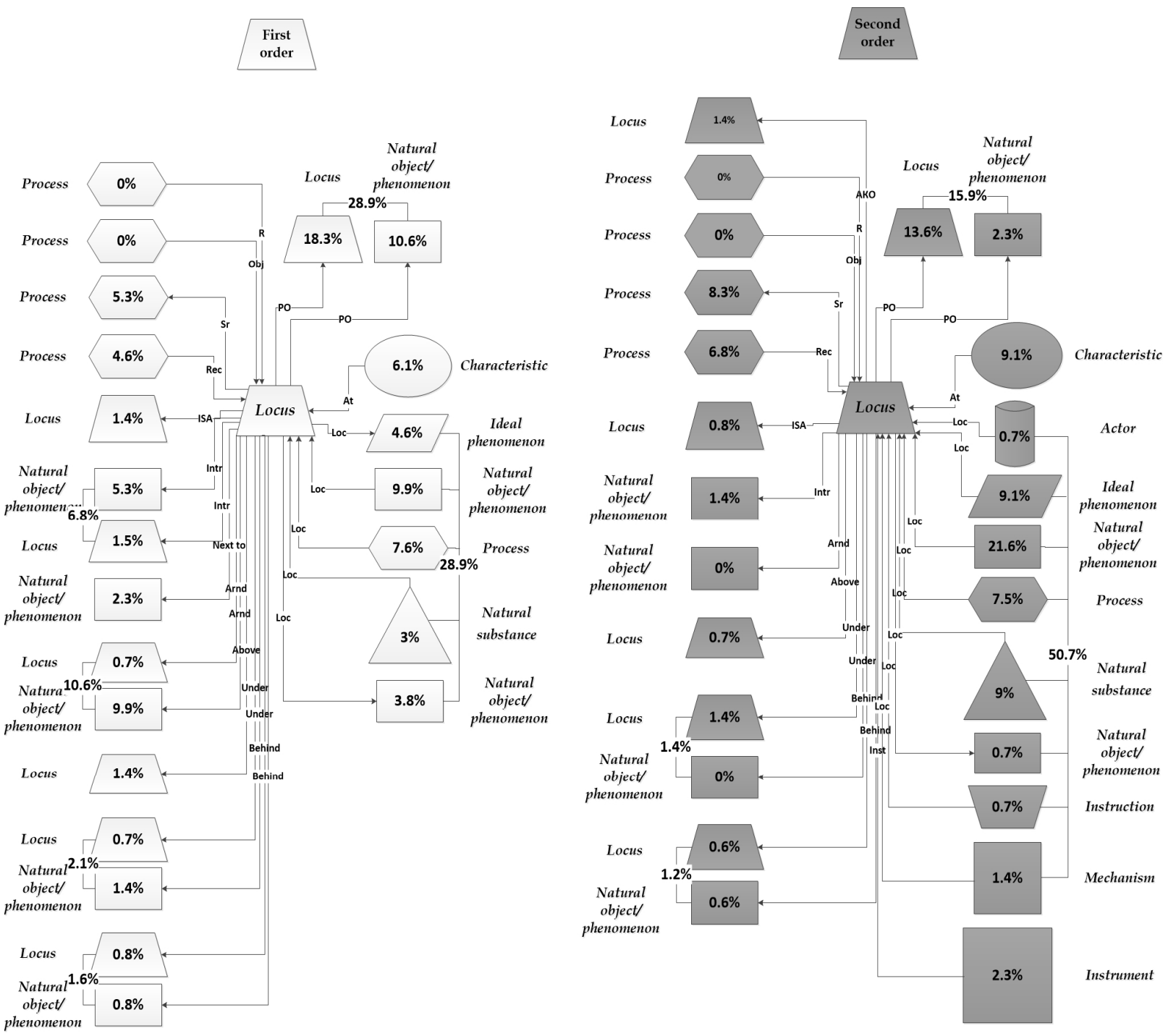


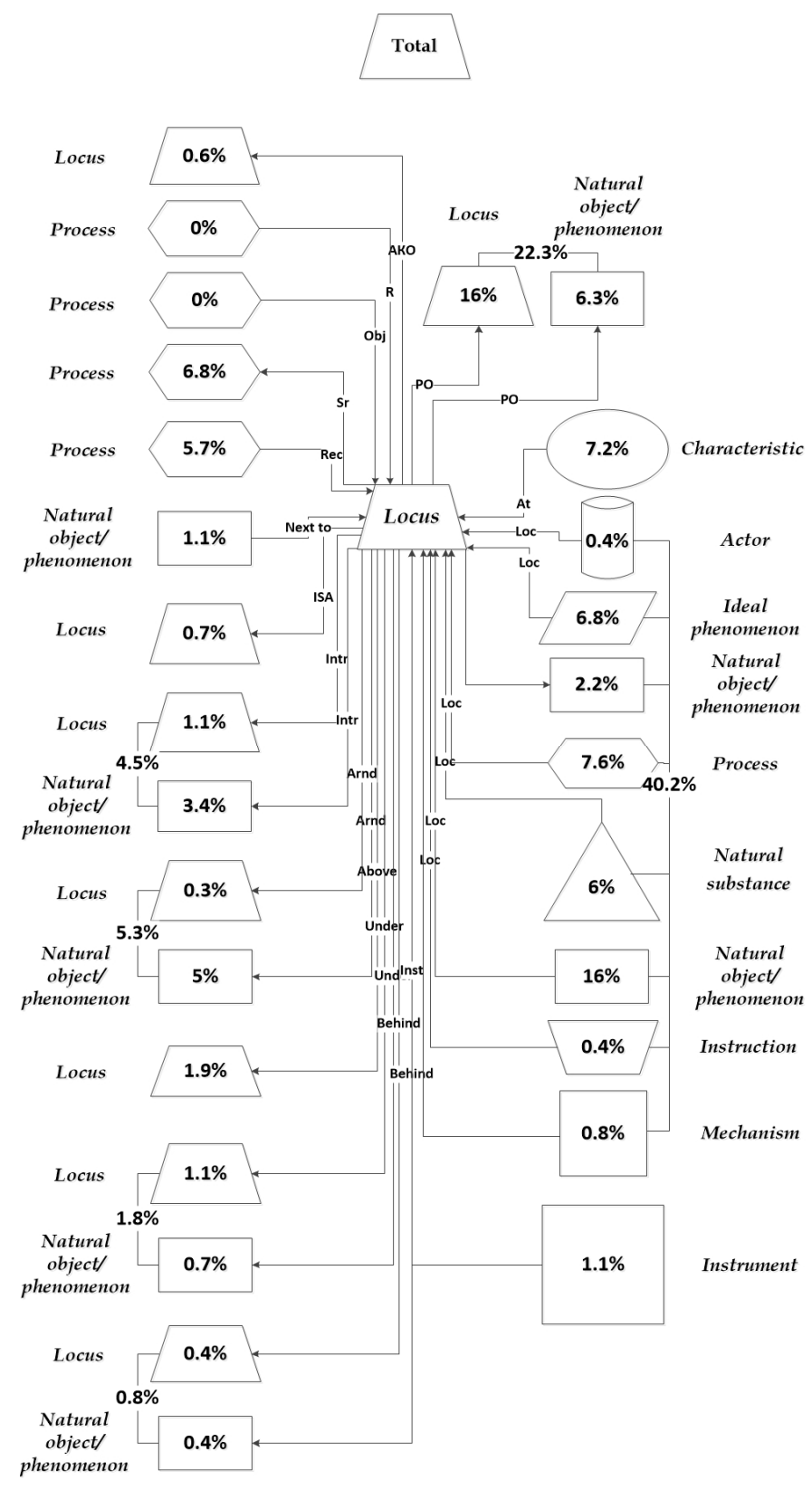

Figure 2. The model of the category Locus interaction with other categories within the terminology based on the results of comparative and statistical analysis

\section{Discussion}

The results of the analysis show that the systemic relations between different categories of technical terms and the category Locus in particular are not just hyponymy relations that form the basis for the terminology networks developed by I. Atanasova (2003), M.G. Malkovskiy (2012) or I.O. Serdceva (2006) and represent a far more diverse set worth noting and studying. Moreover, AKO semantic relation turned to be not so productive for the considered category of technical terms as compared to some other semantic relations such as Loc or PO that appear to be more numerous and frequent. 
The defined patterns illustrated by figure 2 represent a general matrix of the technical terms of the category Locus interaction with the specialized vocabulary units of other categories. The links with certain categories demonstrate the semantic schemes that the category Locus may potentially establish within a terminology system. Nevertheless, the number of such patterns and the adjacent concepts varies in each case. The fact why some semantic schemes are present for one technical term and absent for another as well as the possible difference in number of the adjacent technical terms within one pattern may be explained by the specificity and complexity of the expressed concept, the level of its exploration or some extralinguistic factors related to the development of the field of knowledge that are beyond the scope of this research.

The model also illustrates the difference between the adjacent technical terms of the first and the second order. Thus, as the first order chart shows, PO semantic relation is very productive in defining the concepts of the studied category as well as the links based on prepositions. The adjacent technical terms of some categories such as Actor, Mechanism linked by loc and inst semantic relations, etc. appear as the second order only as they are not used in definitions of the concepts of the category Locus and were extracted from the scientific texts. Thus, the variety of categories linked by loc semantic relation is larger for the second order. The number of the adjacent technical terms of the second order linked to the units of the analyzed category by loc semantic relation is almost twice as many as that of the first order. Consequently, the most productive semantic schemes are those that link the category Locus with the categories Natural object/phenomenon and Process by loc semantic relation and the category Locus with the category Locus by PO semantic relation. The differentiation of vertices and semantic relations as well as correlations between them may also make the automatic processing of inquiries more precise and be considered among other advantages of semantic networks as a tool for the study of terminologies, knowledge representation and knowledge-based systems development discussed by A-B. M. Salem and M. Alfonse (Salem \& Alfonse, 2008). The comparative analysis of the productive semantic schemes of other categories of concepts represents an issue for further studies.

\section{Conclusion}

As the research has revealed, the technical terms of the category Locus are characterized by the specificity of organization and interaction with those of other categories within a terminological network. The semantic relations differ in percentage and the types of concepts they connect to the analyzed category. Loc semantic relation proved to be the most productive as there are usually various referents of different origin that are located or occur within the borders of a definite region in space. This relation has the potential to link the considered category with the largest number of other categories, among which are Process, Natural Object, Substance, Ideal phenomenon, Construction, Mechanism, thus establishing definite semantic schemes. The technical terms that are linked by this relation belong to both the first and the second order. Generally, PO semantic relation is also very productive and is more likely to appear in definitions where technical terms of other categories have AKO semantic relation. In this respect many of the technical terms are of the first order. This semantic relation links the technical terms of the same category Locus. Some regions are defined within space objects the concepts of which are also linked by this semantic relation. At semantic relation links the technical terms of the category Locus to the technical terms of the category Characteristic.

Apart from Loc semantic relation, the technical terms of the category Process can be linked to the considered category by $\mathrm{Sr}$ and Rec semantic relations that are almost equally 
productive. As for the semantic relations based on prepositions, Arnd and Inter are productive. These link the category Locus to the category Natural object/phenomenon. AKO (links to the category Locus), Inst (links to the category Mechanism) semantic relations proved to be unproductive for different reasons. Semantic relations based on prepositions (except for Arnd and Inter) proved to be relatively unproductive as well. These either relate the technical terms of the same category Locus or with the category Natural object/phenomenon. Each of the unproductive semantic relations constitutes less than $4 \%$. Ag and $\mathrm{R}$ semantic relations were not detected at all for the category Locus in the terminological network. This makes it similar to the category Characteristic, but differentiates it from other categories such as Natural object/phenomenon or Mechanism that have the potential to establish them. Nonproductiveness of AKO semantic relation to some extent makes the analyzed category similar to the categories Process and Characteristic. As it has been illustrated, the spread in percentage rate for the productive semantic relations proved to be quite large. A few categories of technical terms have the potential to be related to the category Locus by means of several semantic relations. The most common are Natural object/phenomenon and Process as well as technical terms of the category Locus itself.

\section{Acknowledgement}

The authors express their gratitude to the Ministry of education and science of the Russian Federation that financially supported the study: project No. 14.Z56.16.4913-MK "The integrated cognitive study and development of prototypic technical term definition model based on frame and semantic network analysis"; project No. 34.3234.2017/PCh "Development of linguistic principles of building an expert system for academic knowledge representation based on semantic terminological networks construction".

\section{References}

Anderson, M. (2011). The Sun, Stars, and Galaxies (Solar System), Chicago, USA: Britannica Educational Publishing.

Anderson, R. (2015). The Cosmic Compendium: Interstellar Travel, USA, North Carolina: Lulu Press Inc.

Atanasova, I., Nakov, S. \& Preslav N. (2003). "Bilingual semantic network for Russian and Bulgarian fine art terminologies". Applied linguistics and foreign language teaching. Scientific forum. Humanities, Vol. 3, pp. 222-229, Varna.

Borge-Holthoefer, J. \& Arenas A. (2010) "Semantic networks: structure and dynamics". Entropy, 12,

Cerban, M. (2010). "Types of lexical relations: reiteration". Annals of the University of Craiova.

Cranmer, S. (2002). Coronal holes and the high-speed solar wind. Cambridge: Harvard-Smithsonian Center for Astrophysics.

Cranmer, S. \& Kohl J. (2003). Coronal Holes and Solar Wind Acceleration. Northeast Harbor, Maine: Springer Science \& Business Media.

Darling, D. \& Schulze-Makuch D. (2016). The Extraterrestrial Encyclopedia. Florida, USA: First Edition Design eBook Publishing.

Dehnen, W. \& Read J. (2011). "N-body simulations of gravitational dynamics". The European Physical Journal Plus, 5(126), 1-28. doi: 10.1140/epjp/i2011-11055-3.

Dougherty, M., Esposito, L. \& Krimigis S. (2009). Saturn from Cassini-Huygens. Northeast Harbor, Maine: Springer Science \& Business Media..

Esomba, S. (2012). The book of life, knowledge and confidence. North Carolina. USA: Lulu Press Inc.

Evans, J. (2013). The Solar Corona: Proceedings of International Astronomical Union Symposium. New Mexico, USA, August, 28-30. 
Fellbaum, C. (1998). WordNet: "An electronic lexical database". Cambridge: MIT Press.

Foster, R., Majid, G. \& Cota A. (2009). Solar Energy: "Renewable Energy and the Environment”. Florida, USA: CRC Press.

Golud, L. \& Jay P. (2010). The Solar corona. New York, USA: Cambridge University Press.

Gray, D. (2005). The Observation and Analysis of Stellar Photospheres. New York, USA: Cambridge University Press.

Grinev-Grinevich, S. (2008). Terminology study. Moscow: Academy.

Harrington, B. (2010). "Semantic Network Approach to Measuring Relatedness". COLING 2010, 23rd International Conference on Computational Linguistics, Beijing, China, August, 23-27.

Habing, H. \& Olofsson H. (2004). Asymptotic Giant Branch Stars. Northeast Harbor, Maine: Springer Science \& Business Media.

Hartley, R. \& Barnden J. (1997). "Semantic networks: visualizations of knowledge". Trends in Cognitive Sciences, 1 (5), pp.169-175.

Jager, de C. (2012). Transactions of the International Astronomical Union: "Reports on Astronomy". New York. USA: Springer Science \& Business Media.

Khoo, C. \& Jin-Cheon N. (2006). "Semantic relations in information science". Annual Review of Information Science and Technology, 40 (1), pp. 157-228.

Kimura, H. \& Mann I. (1998). Brightness of the solar F-corona. Katlenburg-Lindau, German.

Kukhno, I. (2014). "Terminology as a semiotic system of the second order". Language. Philology. Culture, 4, pp. 41-55.

Lande, D. (2014). "Building of Networks of Natural Hierarchies of Terms Based on Analysis of Texts Corpora. E-preprint” arXiv 1405.6o68. Retrieved from: http://dblp.org/rec/html/journals/corr/Lande14

Lang, K. (2007). A Companion to Astronomy and Astrophysics. New York. USA: Springer Science \& Business Media.

Latu, M. (2015). Technical Terms Coining in Developing Terminologies. Pyatigorsk: SNEG.

Latu, M. (2016). "Towards the possibility of defining prototype schemes of technical terms relations in semantic networks". Proceedings of the IX International scientific-practical conference, North Charleston, SC, USA, pp. 115-118.

Lieberman, S. (2014). English Learner's Guide to Homophones and Heteronyms. Raleigh, North Carolina, USA: Lulu Press Inc.

Malkovskiy, M. \& Soloviev S. (2003). "Methods of Glossary Formation in the Universal Terminology Space". Computational Linguistics and Smart Technologies. Proceedings of the International Conference, pp. 438-440.

Malkovskiy, M. \& Soloviev S. (2004a). "The Project of a Universal System of Declarative Knowledge Formation". Proceedings of the $1^{\text {st }}$ Cognitive Science Conference, pp. 148-149.

Malkovskiy, M. \& Soloviev S. (2004b). "Structural Method of Inquiry Formation to the Information System. Computational Linguistics and Smart Technologies" Proceedings of the International Conference, Moscow Nauka, pp. 612-613.

Malkovskiy, M. \& Soloviev S. (2012). "Terminological Networks. Open Semantic Technologies of Intellectual Systems Projection”. Proceedings of the II International Scientific Conference, OSTIS, pp. 77-82.

Malkovskiy, M. \& Soloviev S. (2014). "The Principles of Terminological Clusters Formation. Open Semantic Technologies of Intellectual Systems Projection”. Proceedings of the II International Scientific Conference, OSTIS, pp. 169-172.

Moldwin, M. (2008). An introduction to Space Weather. New York, USA: Cambridge University Press.

National Research Council. (2003). Burning Plasma: Bringing a Star to Earth. Washington DC, USA: The National Academies Press.

Pasachoff, J. \& Filippenko A. (2013). The Cosmos: Astronomy in the New Millennium (4Ed.). Cambridge, UK: Cambridge University Press.

Russell, C. (2003). Solar Wind and Interplanetary Magnetic Field: A Tutorial. Los Angles, California: Institute of Geophysics and Planetary Physics and Department of Earth and Space Sciences University of California.

Salem, A-B. \& Alfonse M. (2008). “Ontology versus semantic networks for medical knowledge representation". Proceedings of the $12{ }^{\text {th }}$ WSEAS International Conference on Computers, pp. 769-774. 
Serdceva, I. (2006). "Semantic networks as a structuring tool of computer science terminology database within the scope of the terminology studies discipline". Bulletin of Omsk State Pedagogical University, 1, pp.191-194.

Sobelman, I. (1992). Atomic and Ionic Spectra and Elementary Processes in Plasma. New York, USA: Nova Science Publishers.

Sowa, J. (1998). Semantic networks. Encyclopedia of Artificial Intelligence, S.C. Shapiro (Ed.) New York, USA, Wiley \& Sons, pp.1011-1024.

Sreepat, J. (2013). Fundamentals of Physical Geology. New Delhi, India: Springer Science \& Business Media. Staicu, S. (2010). "Technical vocabulary of medicine". Annals of the University of Craiova, 11 (1). 380 pp.

Steyvers, Mark \& Tenenbaum J. (2005). "The large-scale structure of semantic networks: statistical analysis and a model of semantic growth". Cognitive Science, 29, pp. 41-78.

Wessels, D. (2010). "Issues of lexicology in language for special purposes". Annals of the University of Craiova, 11 (1), pp. 198-213.

Zeilik, M. (2002). Astronomy: The Evolving Universe. Cambridge, UK: Cambridge University Press.

Wikiwand. Corona. Retrieved from on: http://www.wikiwand.com/en/Corona

Internet source: The Stanford Solar Center. The Solar Corona. Retrieved from: http://www.lund.irf.se/HeliosHome/corona.html

Internet source: The free dictionary by Farlex. Retrieved from: http://encyclopedia2.thefreedictionary.com/Roche+lobes 